\title{
ON BINARY MODULAR GROUPS AND THEIR INVARIANTS.
}

BY PROFESSOR L. E. DICKSON.

A VERY simple determination is here made of all groups $G$ of binary linear transformations with integral coefficients of determinant unity modulo $p$ such that the order of the group is divisible* by the prime $p$. The corresponding problem for which the coefficients are in any finite field of order a power of $p$ has been treated recently by $\mathrm{H}$. $\mathrm{H}$. Mitchell, $\uparrow$ who cites the earlier treatments by Gierster, E. H. Moore, Wiman, and Dickson of the related linear fractional group. To be added to these references is a paper on the present binary homogeneous groups. $\ddagger$

Any binary modular transformation $T$ multiplies some linear function of $x$ and $y$ by a constant. This constant is unity if $T$ is of period $p$. Hence after a suitable choice of the variables, we may assume that our group $G$ contains

$$
T: x^{\prime} \equiv x+y, \quad y^{\prime} \equiv y(\bmod p) .
$$

Theorem. Either $G$ is the group $\Gamma$ of all binary transformations with integral coefficients of determinant unity modulo $p$, or else every transformation of $G$ is of the form

$$
x^{\prime} \equiv t x+l y, \quad y^{\prime} \equiv t^{-1} y(\bmod p) .
$$

Suppose that $G$ contains a transformation $R$ for which $y^{\prime}$ involves $x$, so that $y^{\prime}=a(x+k y), a \neq 0$. Then $G$ contains $\S$

$$
P=R^{-1} T^{k}: x^{\prime} \equiv y / a, \quad y^{\prime} \equiv-a x+b y(\bmod p),
$$

in which the value of $b$ is immaterial. Next, $G$ contains

$$
T^{b / a} P: x^{\prime \prime} \equiv y / a, \quad y^{\prime \prime} \equiv-a x .
$$

* The groups of orders prime to $p$ may be found as in the case of binary collineation groups.

† Trans. Amer. Math. Soc., vol. 12 (1911), p. 207.

$\ddagger$ Dickson, "Binary modular groups and their invariants," Amer. Jour. Math., vol. 33 (1911), p. 175 . Here are found the invariants of any modular group other than one composed only of transformations (1), the case treated in the text.

$\$$ To form the product $R P$, we note that $x^{\prime \prime}=a^{-1} y^{\prime}$ under $P$ and eliminate $y^{\prime}$ by means of the equation for $R$. But $x^{\prime \prime} \equiv x+k y$ under $T^{k}$ 。 
The latter transforms $T$ into

$$
x^{\prime} \equiv x, \quad y^{\prime} \equiv y-a^{2} x .
$$

A power of this is $x^{\prime} \equiv x, y^{\prime} \equiv y+x$. The latter and $T$ are known to generate the group $\Gamma$. Hence the theorem is proved.

The group $G$ of transformations (1) is generated by $T$ and

$$
x^{\prime} \equiv \tau x, \quad y^{\prime} \equiv \tau^{-1} y,
$$

where $\tau$ belongs to a certain exponent $d$ modulo $p$. Thus $G$ is of order $p d$.

Evidently $T$ leaves absolutely unaltered the product

$$
\begin{aligned}
\lambda=x(x+y)(x+2 y)+\cdots+ & (x+\overline{p-1} y) \\
& \equiv x^{p}-x y^{p-1}(\bmod p),
\end{aligned}
$$

the congruence holding in view of Fermat's theorem. Now (2) replaces $\lambda$ by $\tau \lambda$. Thus $G$ has the relative invariants $\lambda$ and $y$.

If (1) leaves the point $(x, y)$ unaltered,

$$
t x+l y \equiv \rho x, \quad t^{-1} y \equiv \rho y(\bmod p) .
$$

If these congruences hold identically, $l \equiv 0, t \equiv \pm 1$, and (1) becomes

$$
x^{\prime} \equiv \pm x, \quad y^{\prime} \equiv \pm y .
$$

First, let $d$ be even and $p>2$. Then $G$ contains the two transformations (5), which leave every point unaltered. A point is called special if it can not be transformed by $G$ into $p d / 2$ distinct points, and hence is unaltered by some transformation (1) not of type (5). For such a transformation, equations (4) are not both identities and determine uniquely $x / y$ as an integer modulo $p$, and hence a real special point $(x, y)$. But $(1,0)$ is unaltered by $G$, while the remaining real points $(k, 1)$ are permuted by the powers of $T$. Thus any invariant which vanishes at a special point has the factor $y$ or $\lambda$. An invariant without a factor $y$ or $\lambda$ therefore vanishes at imaginary points falling into sets of $p d / 2$ points conjugate under $G$. Now $y^{p d / 2}$ and $\lambda^{d / 2}$ are unaltered by $T$ and changed in sign by $(2)$, since $\tau^{ \pm d / 2} \equiv-1(\bmod p)$. Hence any linear combi- 
nation of them is an invariant of $G$. We can find* a product of such combinations which has integral coefficients and vanishes at any assigned point, not a special point. Thus the invariant is the product of one or more such products.

For $d$ odd, a non-special point is one of $p d$ conjugates under $G$. We now use the absolute invariants $y^{p d}, \lambda^{d}$.

ThEOREM. As a fundamental system of invariants of a group of transformations (1), we may take $y$ and $\lambda$.

In particular, this theorem yields the seminvariant leaders of invariants of two pairs of cogredient variables.

University of Chicago,

February, 1913.

\title{
ON SOME SYSTEMS OF COLLINEATION GROUPS.
}

\author{
BY DR. HOWARD H. MITCHELL.
}

(Read before the American Mathematical Society, April 26, 1913.)

$\S 1$.

Some systems of collineation groups which arise in connection with the theory of elliptic functions have been investigated by Klein $\nmid$ and Hurwitz $\ddagger$. One of them is a system in $n$ variables each group of which contains an invariant subgroup of order $n^{2}$. For $n$ a prime the quotient group with respect to this invariant subgroup is $(1,1)$ isomorphic with the modular group on two indices of order $n\left(n^{2}-1\right)$. The group in three variables is the Hessian group of order 216.

For $n$ odd there is also an invariant subgroup of order $2 n^{2}$, and there exist two other groups in $(n-1) / 2$ and $(n+1) / 2$ variables each of which is isomorphic with the quotient group with respect to this subgroup. Thus for $n=5$ there is both a binary and a ternary $G_{60}$ and for $n=7$ both a ternary and a quaternary $G_{168}$.

Similar systems of groups in $n^{2},\left(n^{2}-1\right) / 2$, and $\left(n^{2}+1\right) / 2$ variables which arise in the theory of hyperelliptic functions

* Dickson, Trans. Amer. Math. Soc., vol. 12 (1911), p. 4.

$\dagger$ Math. Annalen, vol. 15 (1879), p. 275; also Klein-Fricke, Modulfunktionen (2) 5 .

$\ddagger$ Math. Annalen, vol. 27 (1885), p. 198. 